\title{
Tunable band gap in graphene with a noncentrosymmetric superlattice potential
}

\author{
Rakesh P. Tiwari and D. Stroud \\ Department of Physics, Ohio State University, Columbus, Ohio 43210, USA
}

(Received 30 January 2009; published 29 May 2009)

\begin{abstract}
We show that, if graphene is subjected to the potential from an external superlattice, a band gap develops at the Dirac point provided the superlattice potential has broken inversion symmetry. As numerical example, we calculate the band structure of graphene in the presence of an external potential due to periodically patterned gates arranged in a triangular graphene superlattice (TGS) or a square graphene superlattice with broken inversion symmetry, and find that a band gap is created at the original and, in the case of a TGS, the "second generation" Dirac point. This gap, which extends throughout the superlattice Brillouin zone, can be controlled, in principle, by changing the external potential and the lattice constant of the superlattice. For a square superlattice of lattice-constant $10 \mathrm{~nm}$, we have obtained a gap as large as $65 \mathrm{meV}$, for gate voltages no larger than $1.5 \mathrm{~V}$.
\end{abstract}

DOI: 10.1103/PhysRevB.79.205435

PACS number(s): 73.61.Wp, 73.20.-r, 73.43.Lp, 73.21.Cd

\section{INTRODUCTION}

Ever since the synthesis of high-quality graphene, ${ }^{1}$ there has been tremendous interest in the properties of this singlelayer form of carbon. Graphene has a honeycomb lattice structure, with two atoms per primitive cell and a hexagonal Brillouin zone (BZ). The Fermi-energy $E_{F}$ of homogeneous, neutral graphene lies at the so-called Dirac point, which occurs at high-symmetry points $K$ in the BZ. In fact, there are two inequivalent Dirac-points $\mathbf{K}_{0}$ and $\mathbf{K}_{0}^{\prime}$ with two distinct valleys of excitations. Near the Dirac point, the density of states is linear in $\left|E-E_{F}\right|$ and the spectrum of quasiparticle states is well described by the Dirac equation for massless fermions. Partly as a result of this electronic structure, ${ }^{1-4}$ graphene has many unusual electronic properties, such as a unique type of quantum Hall effect, ${ }^{1,5}$ ballistic conduction by massless Dirac fermions, ${ }^{1,5}$ size-dependent band gap, ${ }^{6}$ large magnetoresistance, ${ }^{4,7,8}$ and gate-tuneable optical transitions. ${ }^{9}$

A number of workers have investigated the possibility of building graphene electronic circuits without physically cutting or etching the graphene monolayer. A natural way to accomplish this is by subjecting graphene to an external potential with a suitable superlattice periodicity, e.g., by applying appropriate gate voltages. Such superlattices have proven to be extremely successful in controlling the electronic structure of more conventional semiconducting materials (see, e.g., Ref. 10). In these materials, the presence of the additional periodic potential gives rise to superlattice electronicband structure, which has extra band gaps at high-symmetry points in the superlattice Brillouin zone (SBZ).

There have been several predictions of electronic effects in graphene due to an external superlattice potential. For example, with one-dimensional (1D) and two-dimensional (2D) superlattices, the group velocity of the low-energy charge carriers is anisotropically renormalized, ${ }^{11}$ while a corrugated graphene sheet is expected to show charge inhomogeneity and localized states. ${ }^{12}$ Superlattices in graphene can be realized experimentally by using periodically patterned gates. Using an electron beam, adatoms with superlattice patterns of periodicity as small as $5 \mathrm{~nm}$ have been achieved on freestanding graphene membranes. ${ }^{13}$ Superlattice patterns have also been observed for graphene on metal surfaces. ${ }^{14-16}$ In a recent theoretical study, a triangular graphene superlattice (TGS) was considered, and a new class of massless Dirac fermions was predicted to occur at the $M$ point in the SBZ. ${ }^{17}$

In the studies so far, the external periodic potential giving rise to the superlattice has had spatial-inversion symmetry. As a result of this symmetry, the degeneracy of the conduction and valence band at the original Dirac point (ODP) is preserved. In the present work, by contrast, we consider the potentials of an external TGS and a square graphene superlattice (SGS) without inversion symmetry. We show that because of the absence of inversion symmetry, an energy gap opens up at both the original and the new Dirac points in the TGS and at the Dirac point in the SGS. The magnitude of these gaps can, in principle, be controlled by modifying the externally applied voltages. Thus far, we have been able to achieve a band gap as large as $65 \mathrm{meV}$ extending throughout the SBZ. Because of the controllability of the band gap, this type of graphene superlattice represents a system in which the band gap can be chosen with considerable freedom by modifying the applied external potential. Such a system, and particularly the control at the ODP, might be quite useful in realizing future graphene electronic circuits.

\section{FORMALISM}

We first describe the formalism we use to calculate the superlattice band structure for a noncentrosymmetric superlattice potential, mostly following the approach of Ref. 17. If the periodicity of the superlattice is much larger than the intercarbon distance $a \sim 1.42 \AA$, which is the case considered here, the intervalley scattering (between $\mathbf{K}_{0}$ and $\mathbf{K}_{0}^{\prime}$ ) can be neglected. ${ }^{18}$ We limit our discussion to the spectrum near one of the two inequivalent Dirac points, which we denote $\mathbf{K}_{0}$, in the presence of a periodic external potential $V(x, y)$. In pure graphene, we use a pseudospin basis $\left(\begin{array}{l}1 \\ 0\end{array}\right) e^{i \mathbf{k} \cdot \mathbf{r}}$ and $\left(\begin{array}{l}0 \\ 1\end{array}\right) e^{i \mathbf{k} \cdot \mathbf{r}}$, where $\left(\begin{array}{l}1 \\ 0\end{array}\right)$ and $\left(\begin{array}{l}0 \\ 1\end{array}\right)$ are Bloch sums of $\pi$ orbitals with wave-vector $\mathbf{K}_{0}$ on sublattices $A$ and $B$, respectively, and $\mathbf{k}$ is the wave vector relative to the $\mathbf{K}_{0}$ point. The single valley Hamiltonian for the quasiparticles of pure graphene is ${ }^{19}$ 


$$
H_{0}=\hbar v_{0}\left(-i \sigma_{x} \partial_{x}-i \sigma_{y} \partial_{y}\right)
$$

where $v_{0}$ is the (isotropic) group velocity at the Dirac point and the $\sigma$ 's are Pauli matrices. The eigenstates and energy eigenvalues are

$$
\psi_{s, \mathbf{k}}^{0}(\mathbf{r})=\frac{1}{\sqrt{2}}\left(\begin{array}{c}
1 \\
s e^{i \theta_{\mathbf{k}}}
\end{array}\right)
$$

and

$$
E_{s}^{0}(\mathbf{k})=s \hbar v_{0} k,
$$

where $s= \pm 1$ is the band index and $\theta_{\mathbf{k}}$ is the polar angle of the wave vector $\mathbf{k}$. Eq. (2) indicates that the pseudospin vector is parallel and antiparallel to $\mathbf{k}$ in the upper $(s=1)$ and lower $(s=-1)$ bands, respectively.

We wish to consider the spectrum of elementary excitations near one of the Dirac points in the presence of $V(x, y)$. The total Hamiltonian then takes the form $H=H_{0}+H^{\prime}$, where

$$
H^{\prime}=I V(x, y),
$$

with $I$ being the $2 \times 2$ identity matrix. We consider a periodic external potential having either a triangular Bravais lattice with basis vectors $\mathbf{b}_{1}=b \hat{x}$ and $\mathbf{b}_{2}=b\left(\frac{1}{2} \hat{x}+\frac{\sqrt{3}}{2} \hat{y}\right)$ or a square Bravais lattice with $\mathbf{b}_{1}=b \hat{x}$ and $\mathbf{b}_{2}=b \hat{y}$. We also assume that $V(x, y)$ varies slowly on the scale of lattice-constant $(b \gg a)$. Then the band structure of the elementary excitations near the $\mathbf{K}_{0}$ point can readily be obtained by diagonalizing $H_{0}$ $+H^{\prime}$ in a plane-wave spinor basis. ${ }^{17}$ The basis states are of the form $\chi(s) \exp [i(\mathbf{k}+\mathbf{G}) \cdot \mathbf{r}]$, where $\chi(s)=\frac{1}{\sqrt{2}}\left(\begin{array}{c}1 \\ e^{i k_{k+G}}\end{array}\right)$ or $\frac{1}{\sqrt{2}}\left(\left(_{-e^{i \theta_{\mathbf{k}+\mathrm{G}}}}\right)\right.$ and $\mathbf{G}$ are reciprocal-lattice vectors corresponding to the periodic external potential $V(x, y)$. If we include $N$ plane waves, we must thus diagonalize a $2 N \times 2 N$ matrix to obtain the band structure of elementary excitations nonperturbatively near the $\mathbf{K}_{0}$ point in the presence of the external periodic potential. We use $N=625$ for both the triangular and the square graphene superlattice. In our actual calculations, we have considered an external potential produced by a periodic array of circular regions, within each of which the potential is a constant. These are easy to treat by the above approach, because the Fourier transform of the constant potential is available analytically. To break the inversion symmetry, we have added a second, smaller circular region within each primitive cell, as illustrated in Figs. 1 and 2. If the second region is not equidistant from the two adjacent larger circular regions, inversion symmetry is broken.

\section{RESULTS}

We first review the case of a triangular array of large circles, with a constant external potential within each circle. Elsewhere in the superlattice, the external potential is zero. The resulting band structure has been computed in Ref. 17 . In the presence of the superlattice, there are several Dirac points: the ODP, which is at $\Gamma$ of the SBZ, and "next generation" Dirac points, at $M$ and $K$ of the SBZ. Of these new Dirac points, only the ODP (with energy set to $0 \mathrm{eV}$ ) and the new Dirac point (NDP) at $M$ with energy $0.196 \mathrm{eV}$ ) have vanishing density of states. ${ }^{17}$ Near both of these points, the

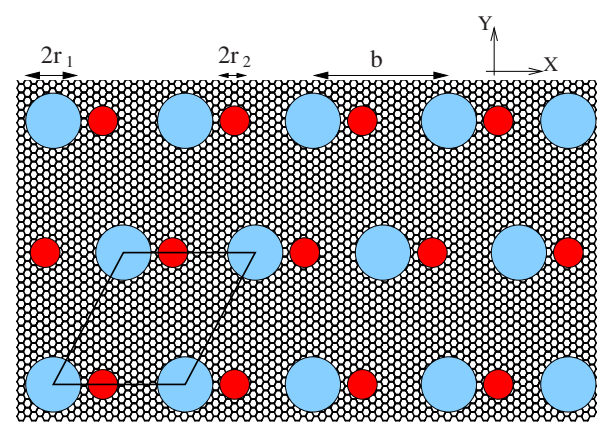

FIG. 1. (Color online) Schematic of graphene plane subjected to a periodic external potential $V(x, y)$ consisting of a periodic arrangement of circular contacts, within each of which $V$ is a constant. The large circles are arranged on a triangular lattice of edge $b$. The small circles are not midway between the large circles; for the case shown, they are centered at $0.4 b \hat{x}$ relative to the centers of the large circles.

density of states goes linearly to zero with energy. However, the group velocity near the NDP is highly anisotropic.

We now consider the superlattice band structure (SBS) for graphene subject to the noncentrosymmetric potential shown in Fig. 1. We choose the lattice constant of the external potential to be $b=10 \mathrm{~nm}$, and the radius $r_{1}$ of the large circles to be $2.5 \mathrm{~nm}$, so that the filling fraction of large circles is 0.226725 . We take the smaller circular gates to have radius $r_{2}=1 \mathrm{~nm}$, and to be centered at $\mathbf{R}+0.4 b \hat{\mathbf{x}}$, where $\mathbf{R}$ is a Bravais vector of the superlattice. The resulting band structure is shown in Fig. 3 for the case $V_{1}=0.5 \mathrm{~V}$ and $V_{2}$ $=-1.65 \mathrm{~V}$, where $V_{1}$ and $V_{2}$ are the voltages on the larger and smaller circles. Once again $V=0$ in the rest of the lattice. A substantial direct gap opens up at the ODP of magnitude $\Delta_{\Gamma}=58.2 \mathrm{meV}$ for these parameters (between the bands above and below the ODP). Although not obvious from Fig. 3 , there is also a gap $\Delta_{M}$ which opens up at the NDP, of magnitude $\sim 1 \mathrm{meV}$. For these parameters, we have checked that there is, in fact, a full band gap at ODP extending through the entire SBZ. The small gap also at $M$ does not extend throughout the SBZ, as is evident from the figure (the gap at $M$ does extend throughout the SBZ for $V_{2}$ positive). The inset of Fig. 3 shows the SBZ, with the symmetry-points $\Gamma, K$, and $M$ indicated.

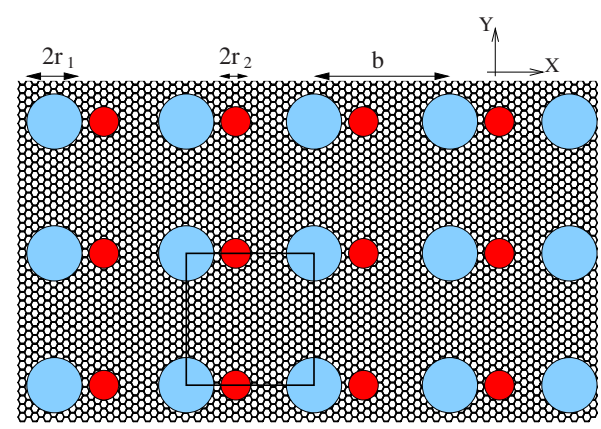

FIG. 2. (Color online) Same as Fig. 1, except that the large and small circular contacts are arranged on a square lattice. Each small circle is now centered at $0.375 b \hat{x}$ relative to the center of the nearest large circle. 


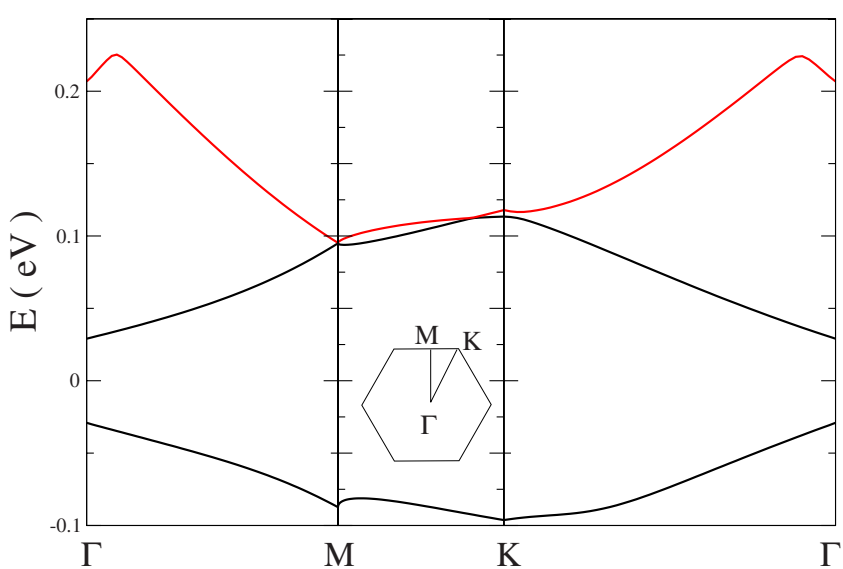

FIG. 3. (Color online) SBS for graphene subjected to an external potential produced by a triangular superlattice of contacts, as shown in Fig. 1. The calculation is carried out using $b=10 \mathrm{~nm}, V_{1}$ $=0.5 \mathrm{~V}, V_{2}=-1.65 \mathrm{~V}, r_{1} / b=0.25, r_{2} / b=0.1$, and the small circles are located at $0.4 \hat{x}$. The $\Gamma$ point of the superlattice corresponds to one of the $K$ points of the original graphene lattice. If only the large circular contacts were present, the SBS would have Dirac points at both $\Gamma$ and $M$ of the SBS Brillouin zone, at both of which the density of states would go linearly to zero. Inset: first Brillouin zone of a triangular lattice showing the three high-symmetry points $\Gamma, M$, and $K$.

In Fig. 4, we show the corresponding SBS for graphene subjected to the periodic potential with square symmetry shown in Fig. 2. Once again, we choose the superlatticeconstant $b=10 \mathrm{~nm}, r_{1}=2.5 \mathrm{~nm}$, and $r_{2}=1 \mathrm{~nm}$ so that the filling fraction of circles in this case is 0.227765 . We choose $V_{1}=0.5 \mathrm{~V}$ and $V_{2}=-1.4 \mathrm{~V}$, and each small circle is located at $0.375 \hat{x}$ relative to the center of each large circle. There is a gap of magnitude $64.4 \mathrm{mV}$, at the ODP at $\Gamma$ which extends throughout the SBZ.

In Fig. 5, we show how the direct gaps at the Dirac point depend on the voltage $V_{2}$ on the smaller circles in the triangular and square superlattices. For the square superlattice, we

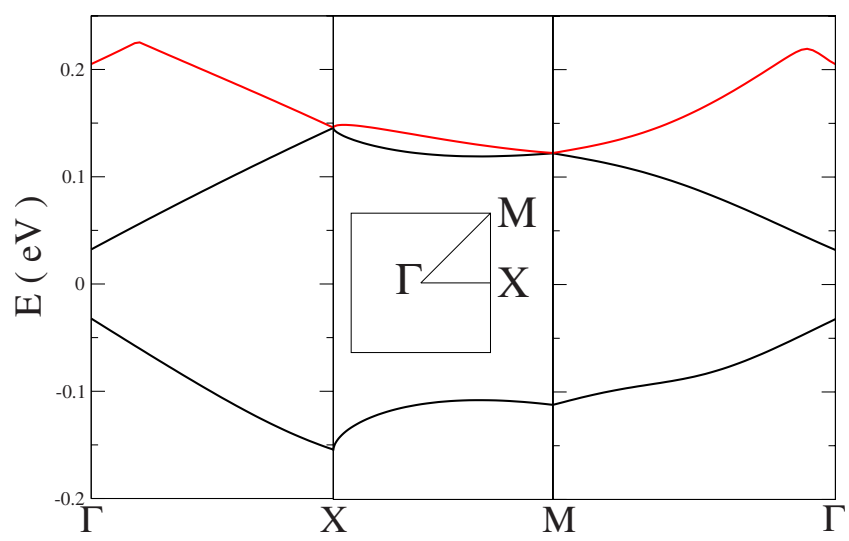

FIG. 4. (Color online) SBS for graphene subjected to an external potential produced by a square superlattice of contacts, as in Fig. 2. The $\Gamma$ point of the superlattice corresponds to one of the $K$ points of the original graphene lattice. In this case, we use $b=10 \mathrm{~nm}, V_{1}$ $=0.5 \mathrm{~V}, V_{2}=-1.4 \mathrm{~V}$, and $r_{1} / b=0.25, r_{2} / b=0.125$, and the small circles are located at $0.375 \hat{x}$. Inset: first Brillouin zone of a square lattice showing the three high-symmetry points $\Gamma, X$, and $M$.

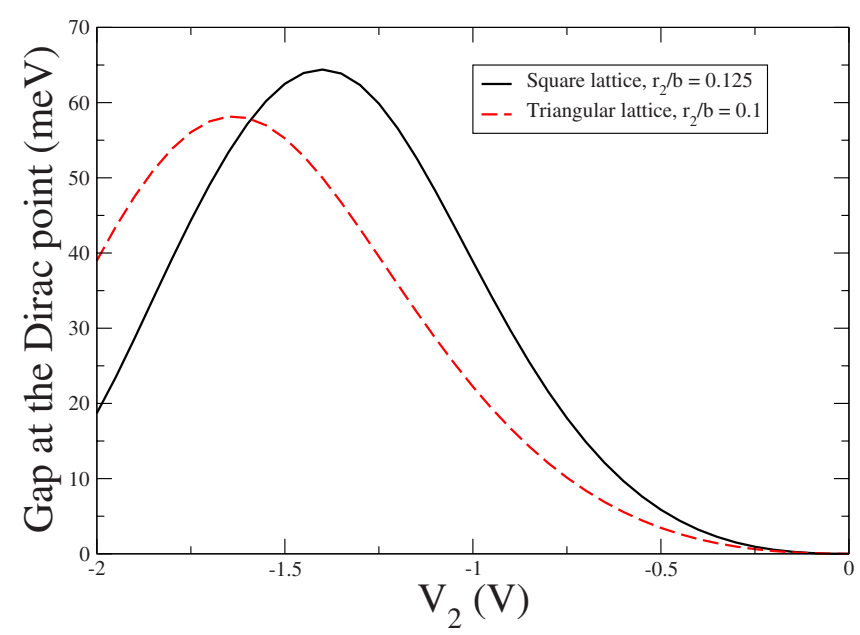

FIG. 5. (Color online) Plot of the gap at the Dirac point $\Gamma$ as a function of the voltage $V_{2}$ (in $V$ ) on the smaller circles. Full curve: square lattice; dashed curve: triangular lattice. For the square lattice, we use $b=10 \mathrm{~nm}, V_{1}=0.5 \mathrm{~V}, r_{1} / b=0.25$, and $r_{2} / b=0.125$, and small circles are located at $0.375 \hat{x}$. For the triangular lattice, $b=10 \mathrm{~nm}, V_{1}=0.5 \mathrm{~V}, r_{1} / b=0.25$, and $r_{2} / b=0.1$, and the small circles are located at $0.4 \hat{x}$.

take $b=10 \mathrm{~nm}, r_{1} / b=0.25 r_{2} / b=0.125$, and $V_{1}$ at $0.5 \mathrm{~V}$, and the small circles are located at $0.375 \hat{x}$. For the triangular superlattice, $b=10 \mathrm{~nm}, r_{1} / b=0.25, r_{2} / b=0.1, V_{1}=0.5 \mathrm{~V}$, and the small circles are located at $0.4 \hat{x}$. In both cases, these gaps show clear maxima as functions of $V_{2}$. We have carried out similar calculations using positive values of $V_{2}$ in both cases and also obtain nonzero gaps extending throughout the SBZ. In the case of positive $V_{2}$, they are generally smaller than for negative voltages.

In Fig. 6, we show the dependence of the gaps at the Dirac point on the radius $r_{2}$ in both the triangular and square superlattices. For the square superlattice, we take $b=10 \mathrm{~nm}$,

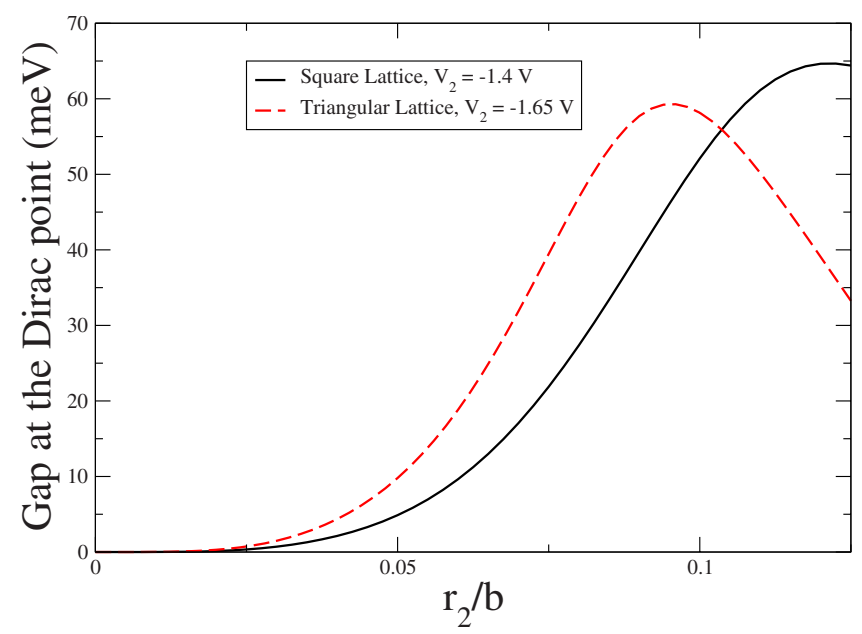

FIG. 6. (Color online) Plot of the gap at the Dirac point as function of $r_{2} / b$. Full curve: square lattice; dashed curve: triangular lattice. For the square lattice, $b=10 \mathrm{~nm}, V_{1}=0.5 \mathrm{~V}, V_{2}=-1.4 \mathrm{~V}$, and $r_{1} / b=0.25$, and small circles are located at $0.375 \hat{x}$. For the triangular lattice, $b=10 \mathrm{~nm}, V_{1}=0.5 \mathrm{~V}$, and $V_{2}=-1.65 \mathrm{~V}, r_{1} / b$ $=0.25$, and small circles are located at $0.4 \hat{x}$. 
$V_{1}=0.5 \mathrm{~V}, V_{2}=-1.4 \mathrm{~V}, r_{1} / b=0.25$ and the small circles are located at $0.375 \hat{x}$. For the triangular case, $b=10 \mathrm{~nm}, V_{1}$ $=0.5 \mathrm{~V}, V_{2}=-1.65 \mathrm{~V}, r_{1} / b=0.25$, and we locate the small circular contacts at $0.4 \hat{x}$. The direct band gaps can reach as large as $\sim 65 \mathrm{meV}$ for the square superlattice.

Because of the special form of the Hamiltonian of Eqs. (1) and (4), the superlattice band structure, and, hence, the band gap at the ODP, exhibit a simple scaling relation: under the transformation $b \rightarrow \lambda b$ and $V(x, y) \rightarrow V(\lambda x, \lambda y) / \lambda$, where $\lambda$ is a dimensionless scaling factor, the eigenvalues of $H$ satisfy $E\left(k_{x}, k_{y}\right) \rightarrow E\left(k_{x} / \lambda, k_{y} / \lambda\right) / \lambda$. Thus, in particular, $\Delta_{\Gamma}$ is multiplied by $1 / \lambda$. For example, if $b$ is halved (and all other lengths are also halved), and the potentials on all the contacts are doubled, then the band gap is also doubled. We have confirmed numerically that this scaling relation is satisfied for our bands.

Besides these calculations, we have tried some other geometries in an effort to obtain the largest possible band gap. For example, we have calculated the SBS for triangular and square superlattices with two off-center circular contacts, one along each edge of the primitive cell. This geometry does not lead to band gaps larger than we have obtained with one off-center circle.

\section{DISCUSSION}

The present results show that the band gap of graphene at the Dirac point can be manipulated by subjecting it to a superlattice potential which lacks inversion symmetry. The magnitude of the band gap can be controlled by modifying the contact potentials. Thus, if such an arrangement of contacts can be created, the resulting material has a band gap which can be controlled via the applied voltages without modifying the structure.

There are several obvious challenges before this scheme could be used in practice. First, the band gaps are rather small (of order $65 \mathrm{meV}$ ). These can be made larger by reducing $b$ while simultaneously increasing $V_{1}$ and $V_{2}$ as implied by the above scaling relation, but this could be challenging experimentally. The gaps can also be increased by increasing the voltages at fixed geometry. Perhaps the most promising scheme might be to increase $b$ while simultaneously increasing the bias voltages. The larger $b$ would be easier to achieve experimentally, while the large voltage offsets would increase the gap. These ideas certainly do not exhaust the possibilities offered by periodic arrangements of contacts. Any 2D superlattice of gates lacking inversion symmetry would lead to a band gap at the ODP, and some which we have not tried may lead to a larger band gap than those we have found to date. A 1D superlattice potential lacking inversion symmetry will not suffice to produce a complete band gap. But such a 1D potential, when combined with a suitable nontime-reversal-invariant perturbation such as a magnetic field, might also lead to a complete band gap.

There are, of course, many other ways to create a band gap in graphene besides the method described here. For example, one could create a noncentrosymmetric lattice of nanoscale holes in graphene. It would be difficult to use this method, however, to create a tunable band gap. In graphene nanoribbons with armchair edges, the band structure becomes insulating if the width of the sample, in units of the lattice-constant $a$, is not of the form $3 M+1$, with $M$ as an integer. $^{20-24}$ But creating such a ribbon requires cutting graphene samples with very high precision, and again, once the ribbon has been created, the band gap cannot be easily tuned. Other possibilities are to induce a gap by using a substrate which lacks inversion symmetry, ${ }^{25}$ or to use graphene antidot lattices. ${ }^{26}$ Again in this case, the gap cannot easily be controlled because the atomic spacing of the substrate is fixed. Our proposal of a TGS (or other 2D superlattice) with a noncentrosymmetric superlattice potential is more efficient in opening up a gap at the ODP. In principle, moreover, this gap can easily be larger than the thermal energy at room temperature for modest values of potential. Thus, this method may be a viable approach to creating graphene with a readily tunable band gap.

In summary, we have calculated the superlattice band structure for graphene subjected to a noncentrosymmetric superlattice of contacts, on each of which the voltage is held constant. We find that the superlattice band structure exhibits a band gap extending throughout the SBZ, which can thus be tuned by external voltages. For some choices of the voltages and superlattice constant, the band gap can be as large as 65 $\mathrm{meV}$, significantly larger than the room-temperature thermal energy. Thus, this arrangement might possibly be of use in future electronic or electromagnetic devices.

\section{ACKNOWLEDGMENTS}

This work has been supported in part by NSF (Grant No. DMR-0820414) through the Materials Research Science and Engineering Center at Ohio State University. We acknowledge valuable conversations with J. P. Pelz.
${ }^{1}$ K. S. Novoselov, A. K. Geim, S. V. Morozov, D. Jiang, M. I. Katsnelson, I. V. Grigorieva, S. V. Dubonos, and A. A. Firsov, Nature (London) 438, 197 (2005).

${ }^{2}$ Y. Zhang, J. W. Tan, H. L. Stormer, and P. Kim, Nature (London) 438, 201 (2005).

${ }^{3}$ C. Berger, Z. Song, X. Li, X. Wu, N. Brown, C. Naud, D. Mayou, T. Li, J. Hass, A. N. Marchenkov, E. H. Conrad, P. N. First, and W. A. de Heer, Science 312, 1191 (2006).
${ }^{4}$ A. K. Geim and K. S. Novoselov, Nature Mater. 6, 183 (2007).

${ }^{5}$ M. S. Purewal, Y. Zhang, and P. Kim, Phys. Status Solidi B 243, 3418 (2006).

${ }^{6}$ M. Y. Han, B. Ozyilmaz, Y. Zhang, and P. Kim, Phys. Rev. Lett. 98, 206805 (2007).

${ }^{7}$ E. W. Hill, A. K. Geim, K. S. Novoselev, F. Schedin, and P. Blake, IEEE Trans. Magn. 42, 2694 (2006).

${ }^{8}$ S. Cho and M. S. Fuhrer, Phys. Rev. B 77, 081402(R) (2008). 
${ }^{9}$ F. Wang, Y. Zhang, C. Tian, C. Girit, A. Zettl, M. Crommie, and Y. R. Shen, Science 320, 206 (2008).

${ }^{10} \mathrm{R}$. Tsu, Superlattice to Nanoelectronics (Elsevier, Oxford, 2005).

${ }^{11}$ C.-H. Park, L. Yang, Y.-W. Son, M. L. Cohen, and S. G. Louie, Nat. Phys. 4, 213 (2008); M. Barbier, F. M. Peeters, P. Vasilopoulos, and J. M. Pereira, Jr., Phys. Rev. B 77, 115446 (2008).

${ }^{12}$ F. Guinea, M. I. Katsnelson, and M. A. H. Vozmediano, Phys. Rev. B 77, 075422 (2008).

${ }^{13}$ J. C. Meyer, C. O. Girit, M. F. Crommie, and A. Zettl, Appl. Phys. Lett. 92, 123110 (2008).

${ }^{14}$ S. Marchini, S. Günther, and J. Wintterlin, Phys. Rev. B 76, 075429 (2007).

${ }^{15}$ A. L. Vazquez de Parga, F. Calleja, B. Borca, M. C. G. Passeggi, Jr., J. J. Hinarejos, F. Guinea, and R. Miranda, Phys. Rev. Lett. 100, 056807 (2008).

${ }^{16}$ Y. Pan, N. Jiang, J. Sun, D. Shi, S. Du, F. Liu, and H. Gao, arXiv:0709.2858 (unpublished).
${ }^{17}$ C.-H. Park, L. Yang, Y.-W. Son, M. L. Cohen, and S. G. Louie, Phys. Rev. Lett. 101, 126804 (2008).

${ }^{18}$ T. Ando and T. Nakanishi, J. Phys. Soc. Jpn. 67, 1704 (1998).

${ }^{19}$ P. R. Wallace, Phys. Rev. 71, 622 (1947).

${ }^{20}$ L. Brey and H. A. Fertig, Phys. Rev. B 73, 235411 (2006).

${ }^{21}$ Y.-W. Son, M. L. Cohen, and S. G. Louie, Phys. Rev. Lett. 97, 216803 (2006).

${ }^{22}$ M. Ezawa, Phys. Rev. B 73, 045432 (2006).

${ }^{23}$ V. Barone, O. Hod, and G. E. Scuseria, Nano Lett. 6, 2748 (2006).

${ }^{24}$ Z. Chen, Y.-M. Lin, M. J. Rooks, and P. Avouris, Physica E (Amsterdam) 40, 228 (2007).

${ }^{25}$ G. Giovannetti, P. A. Khomyakov, G. Brocks, P. J. Kelly, and J. van den Brink, Phys. Rev. B 76, 073103 (2007).

${ }^{26}$ T. G. Pedersen, C. Flindt, J. Pedersen, N. A. Mortensen, A.-P. Jauho, and K. Pedersen, Phys. Rev. Lett. 100, 136804 (2008). 